\title{
Brain and nerves inaugural editorial
}

\author{
Henrique C.S. Muela* \\ Department of Physiology, Agostinho Neto University, Luanda, Angola
}

From the ancient Egyptian mummifications to 18th century scientific research on "globules" and neurons, there is evidence of neuroscience practice throughout the early periods of history. The early civilizations lacked adequate means to obtain knowledge about the human brain. Their assumptions about the inner workings of the mind, therefore, were not accurate. Early views on the function of the brain regarded it to be a form of "cranial stuffing" of sorts.

In the Mediaeval history (Circa 1000-1200), Al-Zahrawi, living in Islamic Iberia, evaluated neurological patients and performed surgical treatments of head injuries, skull fractures, spinal injuries, hydrocephalus, subdural effusions and headache. Concurrently in Persia, Avicenna also presented detailed knowledge about skull fractures and their surgical treatments. Between the 13th and 14th centuries, the first anatomy textbooks in Europe, which included a description of the brain, were written by Mondino de Luzzi and Guido da Vigevano.

Neuroscience during the twentieth century began to be recognized as a distinct unified academic discipline, rather than studies of the nervous system being a factor of science belonging to a variety of disciplines.

The Decade of the Brain (1990-1999) spurred the development of thousands of new findings and dozens of theories about the brain and learning. Two basic types of learning theories were strengthened at this time: modular, domain specific versus global theories. Modular, domain-specific theories mainly focus on explaining the neural mechanisms of skills such as mathematics, reading, attention, and memory. These studies tend to be very precise studies of very specific skills, such as how the brain perceives phonemes, or how a specific aspect of the brain is responsible for human face memories. Global theories of learning provide overarching beliefs about how the brain learns best. Kurt Fischer and others, for example, recognized the value of neuroscience research in education and began to envision an independent field at this time.

As with many scientific fields, the majority of journals publishing neuroscience research are subscription-based. Individuals wanting to read their contents must pay to receive access. A search of PubMed articles using the terms "neuroscience" or "brain" reveals that until 2010 less than $10 \%$ of articles were published in open access journals, or in hybrid subscription journals that offer an open access option, under licenses that allow for redistribution and reuse. The percentage is increasing, but as of 2012 it was only at $18 \%$. So the emergence of open access journals becomes even more important for the sake of scientific knowledge.

Brain and Nerves (JBN) is a bimonthly, open access, peer-reviewed journal which considers manuscripts on all aspects of brain and nerves.

JBN offers an open access platform for publishing the most exciting researches to the authors on various fields including systems neuroscience, cognition and brain diseases. It aims to publish novel work that advances our insight into neurodegeneration mechanisms, neurological diseases and neuropsychiatric disorders, interdisciplinary investigations of nervous system structure and function.

In the best interests of the medical and scientific community, JBN will be freely accessible via the Internet for immediate worldwide, open access to the full text of articles. JBN applies the Creative Commons Attribution License under which anyone is free to copy, distribute, and display the work, provided the original work, first published by JBN, is properly cited. All readers will be able to download and/or print any article at no cost.

I deeply believe that this journal will link one of the world's leading academic medical centers with the international medical community through the shared values enunciated by the JBN.

I close this short message by inviting everyone to submit their exciting research to JBN. All papers receiving a high degree of enthusiasm in the peer-review process will find a home in JBN. Therefore I welcome you to this jornal. With your support as authors, reviewers, and editors, I see very bright prospects for JBN to serve science and the scientific community from all over the world even better in the future. helpful.

Thank you and I hope you will find JBN very informative and

Correspondence to: Henrique Muela, Department of Physiology, Agostinho Neto University, Luanda, Angola, E-mail: henrimuela@hotmail.com

Received: March 10, 2017; Accepted: April 19, 2017; Published: April 21, 2017
Copyright: (C2017 Muela HCS. This is an open-access article distributed under the terms of the Creative Commons Attribution License, which permits unrestricted use, distribution, and reproduction in any medium, provided the original author and source are credited. 